\title{
Global health and chronic non-communicable conditions: Spina bifida care across a worldwide community
}

\author{
Jonathan Castillo* and Heidi Castillo \\ Developmental Pediatrics, Department of Pediatrics, Baylor College of Medicine, Houston, TX, USA
}

Keywords: Global health, blue marble health, chronic non-communicable diseases, spina bifida, international healthcare

\begin{abstract}
"Blue marble health" was introduced in 2013 as a policy framework to illustrate trends in the geographic distribution of neglected tropical diseases affecting vulnerable populations that live not only in low-income countries but also in pockets of the populace in wealthier nations [1]. More recently, inquiry into evidence released by the World Health Organization (WHO) revealed that the concepts of "blue marble health" extend beyond neglected tropical diseases to also include malaria, tuberculosis, and HIV/AIDS [2], all known to cause shuntable hydrocephalus. Concurrently, from the Americas to Europe the prevalence estimates of neural tube defects (NTDs) vary widely even among upper-middle to high-income countries [3]. Though legislation mandating folic acid fortification of staple cereal grains has been introduced throughout many of these countries [4], divergent prevalence rates remain among their populations. Thus, we may wonder if this framework of "blue marble health", which expounds a new global health model replacing the older concept that simply juxtaposed low-income versus high-income countries, also has a role in illustrating chronic non-communicable conditions such as spina bifida (SB).

In this issue of the journal, from the foot of the Andes Mountains, Prado summarizes the efforts in NTD
\end{abstract}

*Corresponding author: Jonathan Castillo, 8080 North Medical Drive, Suite 180, Houston, TX 77054, USA. Tel.: +1 832822 3400; Fax: +1 832825 3399; E-mail: jcporter@ texaschildrens.org. prevention and management of individuals living with SB in Chile. Her summary explains the challenges families face in accessing community-based rehabilitation services and their efforts to form a national family association to foster quality of life and promote social inclusion for its members. She traces the evolution of a program to fortify wheat flour with folic acid. She concludes with a provocative exposition on the advances in genomic testing and the ramifications of the "nonperson" legal status in light of locally available prenatal surgical NTD closure [6].

Throughout several countries in the Americas and Africa, where corn meal and maize flour are used in the preparation of common indigenous meals, these staples have been fortified with at least iron for decades. Current efforts purpose to further support national strategies to increase the supply of micronutrients present in inadequate native diets through evidenceinformed recommendations on the fortification of such corn meal and maize flour with vitamins and minerals [7]. Despite fortification recommendations athwart the globe, rates of new folic acid preventable NTD cases often remain calcitrant to reduction early in the effort [8]. In upper-middle income regions additional activities are at times employed. From the Northern Hemisphere, Martinez de Villarreal in Monterrey, Mexico describes a novel strategy adopted across the region in order to increase folate blood levels among Mexican women. She documents the results of their 
strategy and argues that a personalized program for each population is often needed.

Lastly, from the Latin American metropolis of Buenos Aires with 12 million inhabitants, of whom eighty-five percent are of European origin [9], Valverde provides a sobering description of the challenges many individuals with SB face throughout Argentina. He reports that nearly $40 \%$ of patients were found to have chronic kidney disease when they accessed care. He chronicles the formation of a network of professionals reaching some of the disenfranchised sectors of society. Their use of telemedicine to serve hard to reach subgroups and the advocacy activities before the Argentine ministry of health are also described.

These articles, which were originally presented at the Spina Bifida Association's (SBA) 3rd World Congress on Spina Bifida Research and Care in San Diego in the spring of 2017, underscore the challenges many individuals living with SB face in their local societies. A highlight of the Congress was honoring Dr. Carla Verpoorten, a true pioneer in the care of those living in poverty who are affected by SB and/or hydrocephalus. She was presented with the SBA's Lifetime Achievement Award for her more than 30 year effort to improve care in Europe and Africa. As a neuro-pediatrician based out of the University Hospital Gasthuisberg (K.U. Leuven, Belgium), she is an advocate for the treatment of the neurogenic bladder adapted to the realities of the Global South.

All together in this issue, we reflect on the fact that poverty modulates the care available to individuals living with SB worldwide. Surprisingly, Africa aside, three of the reports stem from nations ranked by the World Bank as upper-middle to high-income economies [10]. Therefore, the tenets of "blue marble health" seem to offer a useful paradigm to continue to explore the factors interrelated to the origins and to the residuum concomitant to NTDs. Furthermore, we also walk away, encouraged by the passion of so many serv- ing with the commitment to an interdisciplinary approach while upholding human dignity. Now it is up to us to heed the call to embark on equitable and sustainable development opportunities for those affected by chronic non-communicable conditions across our diverse and global community.

\section{References}

[1] Hotez PJ, Damania A, Naghavi M. Blue Marble Health and the Global Burden of Disease Study 2013. PLoS Neglected Tropical Diseases. 2016 Oct; 10(10): e0004744.

[2] Hotez PJ. Blue marble health and "the big three diseases": HIV/AIDS, tuberculosis, and malaria. Microbes and Infection. 2015 Aug; 17(8): 539-41.

[3] Zaganjor I, Sekkarie A, Tsang BL, Williams J, Razzaghi H, Mulinare J, et al. Describing the Prevalence of Neural Tube Defects Worldwide: A Systematic Literature Review. PloS One. 2016; 11(4): e0151586.

[4] Crider KS, Bailey LB, Berry RJ. Folic acid food fortificationits history, effect, concerns, and future directions. Nutrients. 2011 Mar; 3(3): 370-84

[5] Warf BC. Hydrocephalus in Uganda: the predominance of infectious origin and primary management with endoscopic third ventriculostomy. Journal of Neurosurgery. 2005 Jan; 102(1 Suppl): 1-15.

[6] Sepulveda W, Wong AE, Sepulveda F, Alcalde JL, Devoto JC, Otayza F. Prenatal diagnosis of spina bifida: from intracranial translucency to intrauterine surgery. Child's Nervous System: ChNS: Official Journal of the International Society for Pediatric Neurosurgery. 2017 Jul; 33(7): 1083-99.

[7] WHO Guideline: Fortification of Maize Flour and Corn Meal with Vitamins and Minerals. Geneva. Switzerland: World Health Organization; 2016.

[8] Botto LD, Lisi A, Robert-Gnansia E, Erickson JD, Vollset SE, Mastroiacovo $\mathrm{P}$, et al. International retrospective cohort study of neural tube defects in relation to folic acid recommendations: are the recommendations working? BMJ. 2005 Mar 12; 330(7491): 571.

[9] Lewis RD. When cultures collide: leading across cultures: a major new edition of the global guide. 3rd ed. Boston; London: Nicholas Brealey International; 2005. xxii, 599 p.

[10] Rank in GDP from world development indicators Database. World Bank; 2016 [9/30/2017]. Available from: http:// databank.worldbank.org/data/download/GDP. 